\title{
Super- and subadditive constructions of aggregation functions
}

\author{
Alexandra Šipošová \\ Department of Mathematics and Descriptive geometry, Faculty of Civil Engineering, \\ Slovak University of Technology, Radlinského 11, 81005 Bratislava, Slovakia \\ E-mail: alexandra.siposova@stuba.sk \\ Ladislav Šipeky \\ Department of Mathematics and Descriptive geometry, Faculty of Civil Engineering, \\ Slovak University of Technology, Radlinského 11, 81005 Bratislava, Slovakia \\ E-mail: ladislav.sipeky@stuba.sk \\ Fabio Rindone \\ Department of Economics and Business \\ University of Catania, 95029 Catania, Italy \\ E-mail: frindone@unict.it \\ Salvatore Greco \\ Department of Economics and Business, \\ University of Catania, Corso, Italia, 55, 95129 Catania, Italy \\ and \\ University of Portsmouth, Portsmouth Business School, \\ Centre of Operations Research and Logistics (CORL), \\ Richmond Building, Portland Street, Portsmouth PO1 3DE, United Kingdom \\ E-mail: salgreco@unict.it \\ Radko Mesiar \\ Department of Mathematics and Descriptive geometry, Faculty of Civil Engineering, \\ Slovak University of Technology, Radlinského 11, 81005 Bratislava, Slovakia \\ and \\ University of Ostrava, IRAFM, 30.dubna 22, Ostrava, Czech Republic \\ E-mail: radko.mesiar@stuba.sk
}

\begin{abstract}
Two construction methods for aggregation functions based on a restricted a priori known decomposition set and decomposition weighing function are introduced and studied. The outgoing aggregation functions are either superadditive or subadditive. Several examples, including illustrative figures, show the potential of the introduced construction methods. Our approach generalizes several known constructions and optimization methods, including decomposition and superdecomposition integrals.
\end{abstract}

Keywords: aggregation function, subadditive transformation, superadditive transformation, decomposition integral. 


\section{Introduction}

Aggregation functions play an important rôle in many domains where an n-dimensional input representation is represented by a single value. For more information and details we recommend monographs [1], [5]. Recall that for $n \in N$ a monotone function $A:[0,1]^{n} \rightarrow[0,1]$ is called an aggregation function whenever it satisfies two boundary conditions $A(0, \ldots, 0)=A(\mathbf{0})=0$ and $A(1, \ldots, 1)=A(\mathbf{1})=1$. Observe that we will not consider the usual convention $A(x)=x$ for 1-dimensional aggregation functions. Note also that, in general, some other interval $I$ can be considered instead of the unit interval $[0,1]$. However, our results related to $[0,1]$ domain can be easily generalized to the domain $I$.

In several practical situations, the aggregation function $A$ is not known on its full domain $[0,1]^{n}$, but only on a subdomain $\mathcal{H} \subseteq[0,1]^{n}$. More often the boundary condition $A(\mathbf{1})=1$ is not important, i.e., $A$ and $\lambda A$ gives the same information for the user, independently of $\lambda \in] 0, \infty[$. This is, e.g., the case when $A$ is considered as a utility function. The above facts have inspired us to introduce two construction methods for aggregation functions when only a partial information is known. Our approach was motivated by the ideas from [6],[7] dealing with superadditive and subadditive transformations of aggregation functions on $\left[0, \infty\left[\right.\right.$. Recall that a function $F:\left[0, \infty\left[^{n} \rightarrow[0, \infty[\right.\right.$ is called superadditive (subadditive) whenever, for any $\mathbf{x}, \mathbf{y} \in\left[0, \infty\left[^{n}\right.\right.$, it holds $F(\mathbf{x}+\mathbf{y}) \geq F(\mathbf{x})+F(\mathbf{y}) \quad(F(\mathbf{x}+\mathbf{y}) \leq$ $F(\mathbf{x})+F(\mathbf{y})) . \quad F$ is additive if and only if it is both superadditive and subadditive, i.e. $F(\mathbf{x}+\mathbf{y})=F(\mathbf{x})+F(\mathbf{y})$. If $F$ is defined on some subdomain $I^{n} \in\left[0, \infty\left[^{n} ;\right.\right.$ then the above inequalities (equalities) are considered for $\mathbf{x}, \mathbf{y} \in I^{n}$ such that also $\mathbf{x}+\mathbf{y} \in I^{n}$.

Our contribution is organized as follows. In Section 2, based on a decomposition set $\mathcal{H}$ and weighing function $B$, we introduce superadditive and subadditive functions $B^{*}$ and $B_{*}$, and the related aggregation functions $A^{\mathcal{H}, B}$ and $A_{\mathcal{H}, B}$, including two motivating examples and some preliminary results. In Section 3, we exemplify the functions $B^{*}$ and $B_{*}$ for several decomposition pairs $(\mathcal{H}, B)$ and show the link of our constructions to decomposition and superdecomposition integrals [9], [10]. Finally, some concluding remarks are added.

\section{Super- and subadditive constructions of aggregation functions}

Fix $n \in N=\{1,2, \ldots\}$. In what follows, an arbitrary subset $\mathcal{H}$ of $[0,1]^{n}$ such that $\mathbf{0} \in \mathcal{H}$ will be called a decomposition set. A function $B: \mathcal{H} \rightarrow[0,1]$, not identically equal to zero, with $B(\mathbf{0})=0$ and such that $B(\mathbf{x}) \leq B(\mathbf{y})$ whenever $\mathbf{x} \leq \mathbf{y}$ for $\mathbf{x}, \mathbf{y} \in \mathcal{H}$, will be called a decomposition weighing function. For any subset $S \subseteq[0, \infty$ [ of non-negative real values, we will denote by inf $S$ the greatest lower bound of $S$, and by sup $S$ the smallest upper bound. If $S$ is unbounded then sup $S=\infty$ by convention. Moreover, the convention that $\inf \emptyset=\infty$ and $\sup \emptyset=0$ will be also considered. 
Although a decomposition weighing function is defined only on $\mathcal{H}$ which, in the extreme case, may consist besides $\mathbf{0}$ just of a single point, one may introduce its transformation to the entire unit $n$-cube $[0,1]^{n}$ by letting

$$
\begin{gathered}
B_{*}(\mathbf{x})=\inf \left\{\sum_{i=1}^{k} B\left(\mathbf{y}^{(i)}\right) \mid k \in N,\left(\mathbf{y}^{(i)}\right)_{i=1}^{k} \in \mathcal{H}^{k} ; \sum_{i=1}^{k} \mathbf{y}^{(i)} \geq \mathbf{x}\right\} \\
\text { and } B^{*}(\mathbf{x})=\sup \left\{\sum_{i=1}^{k} B\left(\mathbf{y}^{(i)}\right) \mid k \in N,\left(\mathbf{y}^{(i)}\right)_{i=1}^{k} \in \mathcal{H}^{k} ; \sum_{i=1}^{k} \mathbf{y}^{(i)} \leq \mathbf{x}\right\} .
\end{gathered}
$$

Observe that, in general, $B_{*}$ and $B^{*}$ are mappings from $[0,1]^{n} \rightarrow[0, \infty]$. The pair $(\mathcal{H}, B)$ will be called subadmissible if $\left.B_{*}(1) \in\right] 0, \infty\left[\right.$, and superadmissible if $\left.B^{*}(1) \in\right] 0, \infty[$. The set of all subadmissible and superadmissible pairs will be denoted simply by $S u b_{n}$ and Super $_{n}$, respectively.

For any subadmissible (superadmissible) pair $(\mathcal{H}, B)$ we may introduce normalized versions of the transformation of $B$ introduced above by letting

$$
A_{\mathcal{H}, B}:[0,1]^{n} \rightarrow[0,1] ; \mathbf{x} \mapsto B_{*}(\mathbf{x}) / B_{*}(\mathbf{1})
$$

and

$$
A^{\mathcal{H}, B}:[0,1]^{n} \rightarrow[0,1] ; \mathbf{x} \mapsto B^{*}(\mathbf{x}) / B^{*}(\mathbf{1}),
$$

where in both cases $\mathbf{1} \in[0,1]^{n}$ denotes the all-one vector.

Let us give two economic examples of possible applications of normalized subadmissible and superadmissible normalized transformations $A_{\mathcal{H}, B}$ and $A^{\mathcal{H}, B}$.

Example 1. Let us suppose that function $B$ is a production function (see e.g. [4, 11]) related to a given product so that from the vector of input quantities $\mathbf{x}=\left[x_{1}, \ldots, x_{n}\right] \in \Re_{+}^{n}$ the quantity $B(\mathbf{x}) \in \Re$ is obtained. More precisely, one can imagine that there is a set of admissible input vectors $\mathcal{H} \subseteq \Re_{+}^{n}$, so that, in fact, one can imagine the production function as mapping from $\mathcal{H}$ to $\Re_{+}$. One can also suppose that the input quantities are normalized so that $\mathbf{x} \in[0,1]^{n}$ and, consequently, $\mathcal{H} \subseteq[0,1]^{n}$. Also the output can be normalized in the interval $[0,1]$. Considering that it could be possible to get a greater output by splitting the production related to a vector of input $\mathbf{x}=\left[x_{1}, \ldots, x_{n}\right] \in[0,1]$ in the family of vector of inputs $\mathbf{y}^{(i)} \in \mathcal{H}^{k}, i=1, \ldots, k$ with $\sum_{i=1}^{k} \mathbf{y}^{(i)} \leq \mathbf{x}$ obtaining as output $\sum_{i=1}^{k} B\left(\mathbf{y}^{(i)}\right)$, by means of the superadditive transformation we get that the maximal output is given by $B^{*}(\mathbf{1})$. Therefore, the normalized production function related to basic production function $B$ and to the set of admissible input vectors $\mathcal{H}$ is given by $A^{\mathcal{H}, B}=B^{*}(\mathbf{x}) / B^{*}(\mathbf{1})$.

Example 2. Let us consider a financial market (see e.g. [3]) where uncertainty is represented by a set of states $S=\left\{s_{1}, \ldots, s_{n}\right\}$. States from $S$ are exhaustive and mutually exclusive so that only one state will be true. In this context each vector $\mathbf{x}=\left[x_{1}, \ldots, x_{n}\right] \in \Re_{+}^{n}$ can be considered as a feasible security that pays an outcome $x_{i}, i=1, \ldots, n$, if the state $s_{i}$ 
is revealed true. Suppose that on the market a set of securities $\mathcal{H} \subset \Re_{+}^{n}$ is available. In this context $B: \mathcal{H} \rightarrow \Re_{+}$is a price function. Fix a vector of outcomes $\mathbf{x}=\left[x_{1}, \ldots, x_{n}\right] \in \Re_{+}^{n}$. A super-replication portfolio ([2]) is a set of securities $\mathbf{y}^{(i)} \in \mathcal{H}, i=1, \ldots, k$, such that $\sum_{i=1}^{k} \mathbf{y}^{(i)} \geq \mathbf{x}$. Among all the super-replication portfolios, one economic operators look for that one with the minimum price which is given by $B_{*}(\mathbf{x})$. One can suppose that all outcomes of considered securities can be normalized so that they take value in $[0,1]$, and one has $B:[0,1]^{n} \rightarrow[0,1]$. Also the prices can be normalized in the interval $[0,1]$. In fact, in this context the maximal attainable vector of outcomes is $\mathbf{1}$ having $B^{*}(\mathbf{1})$ as minimal price of the super replication portfolio. Therefore the prices of portfolio $\mathbf{x} \in[0,1]^{n}$ in the considered financial market is given by $A_{\mathcal{H}, B}=B_{*}(\mathbf{x}) / B_{*}(\mathbf{1})$.

Quite expectedly, the introduced functions $B_{*}$ and $B^{*}$ as well as their normalized versions $A_{\mathcal{H}, B}$ and $A^{\mathcal{H}, B}$, are subadditive and superadditive, respectively:

Proposition 1 If $(\mathcal{H}, B)$ is a subadmissible pair, then $A_{\mathcal{H}, B}$ is a subadditive aggregation function. Analogously, if $(\mathcal{H}, B)$ is a superadmissible pair, then $A^{\mathcal{H}, B}$ is a superadditive aggregation function.

Proof. Because of subadmissibility and superadmissibility assumptions, the functions $A_{\mathcal{H}, B}$ and $A^{\mathcal{H}, B}$ are well defined. Monotonicity of both $A_{\mathcal{H}, B}$ and $A^{\mathcal{H}, B}$ follow from the monotonicity and non-negativity of decomposition weighing functions. Clearly, $A_{\mathcal{H}, B}(\mathbf{0})=$ $\mathbf{0}\left(A^{\mathcal{H}, B}(\mathbf{0})=\mathbf{0}\right)$ and $A_{\mathcal{H}, B}(\mathbf{1})=\mathbf{1}\left(A^{\mathcal{H}, B}(\mathbf{1})=\mathbf{1}\right)$. It remains to prove sub- and superadditivity, and it is clearly sufficient to do this for $B_{*}$ and $B^{*}$.

For arbitrary $\mathbf{x}, \mathbf{y} \in[0,1]^{n}$ let $\left(\overline{\mathbf{x}}^{(i)}\right)_{i=1}^{k}$ and $\left(\overline{\mathbf{y}}^{(j)}\right)_{j=1}^{\ell}$ be a $k$-tuple and an $\ell$-tuple of vectors in $\mathcal{H}$ for which $\sum_{i=1}^{k} \overline{\mathbf{x}}^{(i)} \geq \mathbf{x}$ and $\sum_{j=1}^{\ell} \overline{\mathbf{y}}^{(j)} \geq \mathbf{y}$. Since, by the choice of these $k$ and $\ell$-tuples, the sum of the vectors in the $(k+\ell)$-tuple $\left(\overline{\mathbf{x}}^{(1)}, \ldots, \overline{\mathbf{x}}^{(k)}, \overline{\mathbf{y}}^{(1)}, \ldots, \overline{\mathbf{y}}^{(\ell)}\right)$ is at least $\mathbf{x}+\mathbf{y}$, it follows by the definition of $B_{*}$ that

$$
B_{*}(\mathbf{x}+\mathbf{y}) \leq \sum_{i=1}^{k} B\left(\overline{\mathbf{x}}^{(i)}\right)+\sum_{j=1}^{\ell} B\left(\overline{\mathbf{y}}^{(j)}\right) .
$$

Now, it is evident that $B_{*}(x+y) \leq B_{*}(\mathbf{x})+B_{*}(\mathbf{y})$.

Similarly, for any $\mathbf{x}, \mathbf{y} \in[0,1]^{n}$ let $\left(\overline{\mathbf{x}}^{(i)}\right)_{i=1}^{k}$ and $\left(\overline{\mathbf{y}}^{(j)}\right)_{j=1}^{\ell}$ be a $k$-tuple and an $\ell$-tuple of vectors in $\mathcal{H}$ for which $\sum_{i=1}^{k} \overline{\mathbf{x}}^{(i)} \leq \mathbf{x}$ and $\sum_{j=1}^{\ell} \overline{\mathbf{y}}^{(j)} \leq \mathbf{y}$. By the choice of these $k$ - and $\ell$-tuples, the sum of the vectors in the $(k+\ell)$-tuple $\left(\overline{\mathbf{x}}^{(1)}, \ldots, \overline{\mathbf{x}}^{(k)}, \overline{\mathbf{y}}^{(1)}, \ldots, \overline{\mathbf{y}}^{(\ell)}\right)$ is this time at most $\mathbf{x}+\mathbf{y}$, and so from the definition of $B^{*}$ we have

$$
B^{*}(\mathbf{x}+\mathbf{y}) \geq \sum_{i=1}^{k} B\left(\overline{\mathbf{x}}^{(i)}\right)+\sum_{j=1}^{\ell} B\left(\overline{\mathbf{y}}^{(j)}\right) .
$$

Again, it is evident that $B^{*}(x+y) \geq B^{*}(\mathbf{x})+B^{*}(\mathbf{y})$. This implies sub- and superadditivity of $B_{*}$ and $B^{*}$ and completes the proof. 
We illustrate our proposals in the next simple example. Let $n=1$ and consider a trivial decomposition system $\mathcal{H}=\{0,1 / t\}$ for some fixed positive integer $t$. Further, let $B$ be a decomposition weighing function defined by $B(0)=0$ and $B(1 / t)=b>0$. Obviously, $B_{*}(0)=0$. For any $\left.\left.x \in\right] 0,1\right]$, letting $k=\lceil t x\rceil$ (the ceiling of $t x$ ) we have $\left.\left.x \in\right](k-1) / t, k / t\right]$, so that $B_{*}(x)=k b$ and hence $B_{*}(1)=t b$; it follows that $A_{\mathcal{H}, B}(x)=B_{*}(x) / B_{*}(1)=\lceil t x\rceil / t$, which is a subadditive aggregation function. By the same token, letting $\ell=\lfloor t x\rfloor$ (the floor of $t x)$ we have $x \in\left[\ell / t,(\ell+1) / t\left[\right.\right.$, so that $B^{*}(x)=\ell b, B^{*}(1)=t b$, and $A^{\mathcal{H}, B}(x)=$ $B^{*}(x) / B^{*}(1)=\lfloor t x\rfloor / t$, which is a superadditive aggregation function.

Proposition 2 If $(\mathcal{H}, B)$ is a subadmissible pair, then $A_{\mathcal{H}, B}=B$ if and only if $\mathcal{H}=[0,1]^{n}$ and $B$ is subadditive, with $B(\mathbf{1})=1$. Analogously, if $(\mathcal{H}, B)$ is a superadmissible pair, then $A^{\mathcal{H}, B}=B$ if and only if $\mathcal{H}=[0,1]^{n}$ and $B$ is superadditive, with $B(\mathbf{1})=1$.

Proof. Since the functions $A_{\mathcal{H}, B}$ and $A^{\mathcal{H}, B}$ are defined on $[0,1]^{n}$ and are sub- and superadditive by Proposition 1 , the conditions $A_{\mathcal{H}, B}=B$ and $A^{\mathcal{H}, B}=B$ imply that $\mathcal{H}=[0,1]^{n}$ and $B$ is subadditive and superadditive, respectively, and $B(\mathbf{1})=1$.

Conversely, if $\mathcal{H}=[0,1]^{n}$ and $B$ is subadditive, then $B_{*}=B$ by [6] and since $1=$ $B(\mathbf{1})=B_{*}(\mathbf{1})$, we have $A_{\mathcal{H}, B}=B$. The proof for the superadditive case is similar and therefore omitted.

Observe that on the space of subadmissible pairs $S u b_{n}$ we have a natural partial order $\varliminf_{S u b}$ defined by

$$
\left(\mathcal{H}_{1}, B_{1}\right) \preceq_{S u b}\left(\mathcal{H}_{2}, B_{2}\right) \text { if and only if } \mathcal{H}_{1} \supseteq \mathcal{H}_{2} \text { and } B_{1} \mid \mathcal{H}_{2} \leq B_{2} \text {. }
$$

Similarly, on the space of superadmissible pairs $\operatorname{Super}_{n}$ we have a natural partial order $\preceq_{\text {Super }}$ defined by

$$
\left(\mathcal{H}_{1}, B_{1}\right) \preceq_{\text {Super }}\left(\mathcal{H}_{2}, B_{2}\right) \text { if and only if } \mathcal{H}_{1} \subseteq \mathcal{H}_{2} \text { and } B_{1} \leq B_{2} \mid \mathcal{H}_{1}
$$

This allows us to compare the values of the corresponding aggregation functions as follows.

Proposition 3 Let $\left(\mathcal{H}_{1}, B_{1}\right),\left(\mathcal{H}_{2}, B_{2}\right) \in S u b_{n}$ and $\left(\mathcal{H}_{1}, B_{1}\right) \preceq_{S u b}\left(\mathcal{H}_{2}, B_{2}\right)$. If $\left(B_{1}\right)_{*}(\mathbf{1})=$ $\left(B_{2}\right)_{*}(\mathbf{1})$, then $A_{\mathcal{H}_{1}, B_{1}} \leq A_{\mathcal{H}_{2}, B_{2}}$. Analogously, if $\left(\mathcal{H}_{1}, B_{1}\right),\left(\mathcal{H}_{2}, B_{2}\right) \in$ Super $_{n}$ are such that $\left(\mathcal{H}_{1}, B_{1}\right) \preceq_{\text {Super }}\left(\mathcal{H}_{2}, B_{2}\right)$ and $B_{1}^{*}(\mathbf{1})=B_{2}^{*}(\mathbf{1})$, then $A^{\mathcal{H}_{1}, B_{1}} \leq A^{\mathcal{H}_{2}, B_{2}}$.

Proof. For a fixed $\mathbf{x} \in[0,1]^{n}$, let $\left(\overline{\mathbf{x}}^{(i)}\right)_{i=1}^{k}$ be a $k$-tuple of vectors in $\mathcal{H}_{2}$ such that $\sum_{i=1}^{k} \overline{\mathbf{x}}^{(i)} \geq \mathbf{x}$. Due to $\left(\mathcal{H}_{1}, B_{1}\right) \preceq_{S u b}\left(\mathcal{H}_{2}, B_{2}\right)$ it holds $\sum_{i=1}^{k} B_{1}\left(\overline{\mathbf{x}}^{(i)}\right) \leq \sum_{i=1}^{k} B_{2}\left(\overline{\mathbf{x}}^{(i)}\right)$, and thus also $\left(B_{1}\right)_{*}(\mathbf{x}) \leq\left(B_{2}\right)_{*}(\mathbf{x})$. Since we have assumed that $\left(B_{1}\right)_{*}(\mathbf{1})=\left(B_{2}\right)_{*}(\mathbf{1})$, it follows that

$$
A_{\mathcal{H}_{1}, B_{1}}(\mathbf{x})=\left(B_{1}\right)_{*}(\mathbf{x}) /\left(B_{1}\right)_{*}(\mathbf{1}) \leq\left(B_{2}\right)_{*}(\mathbf{x}) /\left(B_{2}\right)_{*}(\mathbf{1})=A_{\mathcal{H}_{2}, B_{2}}(\mathbf{x})
$$


Similarly, if $\left(\overline{\mathbf{x}}^{(i)}\right)_{i=1}^{k}$ is a $k$-tuple of vectors in $\mathcal{H}_{1}$ such that $\sum_{i=1}^{k} \overline{\mathbf{x}}^{(i)} \leq \mathbf{x}$, the assumption $\left(\mathcal{H}_{1}, B_{1}\right) \preceq_{\text {Super }}\left(\mathcal{H}_{2}, B_{2}\right)$ ensures that $\sum_{i=1}^{k} B_{1}\left(\overline{\mathbf{x}}^{(i)}\right) \leq \sum_{i=1}^{k} B_{2}\left(\overline{\mathbf{x}}^{(i)}\right)$, and thus also $B_{1}^{*}(\mathbf{x}) \leq B_{2}^{*}(\mathbf{x})$. Now, from $B_{1}^{*}(\mathbf{1})=B_{2}^{*}(\mathbf{1})$ it follows that

$$
A^{\mathcal{H}_{1}, B_{1}}(\mathbf{x})=B_{1}^{*}(\mathbf{x}) / B_{1}^{*}(\mathbf{1}) \leq B_{1}^{*}(\mathbf{x}) / B_{1}^{*}(\mathbf{1})=A^{\mathcal{H}_{2}, B_{2}}(\mathbf{x}),
$$

which completes the proof.

Remark. The above result will not be valid in general if, say, in the $\operatorname{Super}_{n}$ case, the assumption $B_{1}^{*}(\mathbf{1})=B_{2}^{*}(\mathbf{1})$ is dropped. To see this, for $n=1, \mathcal{H}_{1}=\{0,1 / 2\}, \mathcal{H}_{2}=$ $\{0,1 / 2,1\}, B_{1}(1 / 2)=1$ and $B_{2}(1 / 2)=1, B_{2}(1)=4$, so that $B_{1}^{*}(1)=2$ and $B_{2}^{*}(1)=4$. It is then easy to see that, for example, $A^{\mathcal{H}_{1}, B_{1}}(1 / 2)=1 / 2$, while $A^{\mathcal{H}_{2}, B_{2}}(1 / 2)=1 / 4$, violating the inequality $A^{\mathcal{H}_{1}, B_{1}} \leq A^{\mathcal{H}_{2}, B_{2}}$.

We continue with an auxiliary result in dimension 1.

Proposition 4 Let $\mathcal{H} \subseteq[0,1]$ be a decomposition set and let $B: \mathcal{H} \rightarrow[0,1]$ be a decomposition weighing function of dimension 1 . Then,

(a) $(\mathcal{H}, B) \in S u b_{1}$ if and only if $\inf \{B(x) / x \mid x \in \mathcal{H} \backslash\{0\}\}>0$, and

(b) $(\mathcal{H}, B) \in$ Super $_{1}$ if and only if $\sup \{B(x) / x \mid x \in \mathcal{H} \backslash\{0\}\}<\infty$.

Proof. We have $(\mathcal{H}, B) \in S u b_{1}$ if and only if $B_{*}(1)>0$, and $(\mathcal{H}, B) \in$ Super $_{1}$ if and only if $B^{*}(1)<\infty$. We begin by proving that $\inf \{B(x) / x \mid x \in \mathcal{H}\}>0$ implies $B_{*}(1)>0$. Suppose that $\inf \{B(x) / x \mid x \in \mathcal{H}\}=b>0$. This means that $B(x) \geq b x$ for every $x \in \mathcal{H}$. Thus, for every $n$-tuple $x_{1}, x_{2}, \ldots, x_{n} \in \mathcal{H}$ such that $\sum_{i=1}^{n} x_{i} \geq 1$ we have $\sum_{i=1}^{n} B\left(x_{i}\right) \geq b \sum_{i=1}^{n} x_{i} \geq b$, so that $B_{*}(1) \geq b>0$. Similarly, assume that $\sup \{B(x) / x \mid x \in \mathcal{H}\}=b<\infty$. This means that $B(x) \leq b x$ for every $x \in \mathcal{H}$. Thus, for every $n$-tuple $x_{1}, x_{2}, \ldots, x_{n} \in \mathcal{H}$ with $\sum_{i=1}^{n} x_{i} \leq 1$ we have $\sum_{i=1}^{n} B\left(x_{i}\right) \leq b \sum_{i=1}^{n} x_{i} \leq b$, implying that $B^{*}(1) \leq b<\infty$.

Conversely, suppose that $\inf \{B(x) / x \mid x \in \mathcal{H}\}=0$. If $B(z)=0$ for some $z \in \mathcal{H}$, then we clearly have $B_{*}(1)=0$. We therefore may assume that $B(x) \neq 0$ for every $x \in \mathcal{H}$. Since $B$ is non-decreasing and positive, the equality $\inf \{B(x) / x \mid x \in \mathcal{H}\}=0$ holds for such a $B$ if and only if there is a sequence $x_{1}, x_{2}, \ldots, x_{n}, \ldots$ of elements of $\mathcal{H}$ such that $\lim _{n \rightarrow \infty} x_{n}=0$ and $\lim _{n \rightarrow \infty} B\left(x_{n}\right) / x_{n}=0$. This means that for every arbitrarily small $\varepsilon>0$ there exists an $n_{\varepsilon}$ such that for every $n \geq n_{\varepsilon}$ we have $B\left(x_{n}\right) \leq \varepsilon x_{n}$. Let $m=\left\lceil 1 / x_{n}\right\rceil$; note that $m-1<1 / x_{n} \leq m$. Since $m x_{n} \geq 1$, for $n \geq n_{\varepsilon}$ we have

$$
B_{*}(1) \leq m B\left(x_{n}\right) \leq \varepsilon m x_{n}<\varepsilon\left(1+1 / x_{n}\right) x_{n}=\varepsilon\left(1+x_{n}\right) \leq 2 \varepsilon
$$

which means that $B^{*}(1)<2 \varepsilon$ for every $\varepsilon>0$ and hence $B_{*}(1)=0$.

It remains to prove the converse in the supremum case. Suppose that $\sup \{B(x) / x \mid x \in$ $\mathcal{H}\}=\infty$; since $B$ is non-decreasing, the only way the supremum attains the value of infinity is that there is a sequence $x_{1}, x_{2}, \ldots, x_{n}, \ldots$ of elements of $\mathcal{H}$ such that $\lim _{n \rightarrow \infty} x_{n}=0$ and $\lim _{n \rightarrow \infty} B\left(x_{n}\right) / x_{n}=\infty$. This means that for every arbitrarily large $k>0$ there exists 
an $n_{k}$ such that for every $n \geq n_{k}$ we have $x_{n} \leq 1 / 2$ and $B\left(x_{n}\right) \geq k x_{n}$. This time let $m=\left\lfloor 1 / x_{n}\right\rfloor$; note that $m \leq 1 / x_{n}<m+1$. Since $m x_{n} \leq 1$, for $n \geq n_{k}$ we have

$$
B^{*}(1) \geq m B\left(x_{n}\right) \geq k m x_{n}>k\left(1 / x_{n}-1\right) x_{n}=k\left(1-x_{n}\right) \geq k / 2
$$

which means that $B^{*}(1)>k / 2$ for arbitrarily large $k>0$ and so $B_{*}(1)=0$. The proof is complete.

Based on Proposition 4, we prove the following general result for any dimension. For a vector $\mathbf{x}=\left(x_{1}, \ldots, x_{n}\right) \in[0,1]^{n}$ we define $\max (\mathbf{x})=\max \left\{x_{i} \mid 1 \leq i \leq n\right\}$ and let $(\mathbf{x})_{i}=x_{i}$ for $1 \leq i \leq n$.

Proposition 5 Let $\mathcal{H} \subset[0,1]^{n}$ be a decomposition set and let $B: \mathcal{H} \rightarrow[0,1]$ be a decomposition weighing function of dimension $n \geq 1$. Then,

(a) $(\mathcal{H}, B) \in S u b_{n}$ if and only if for each $i \in\{1, \ldots, n\}$ there is an $\mathbf{x} \in \mathcal{H}$ such that $(\mathbf{x})_{i}>0$ and $\inf \left\{\frac{B(\mathbf{x})}{(\mathbf{x})_{i}} \mid \mathbf{x} \in \mathcal{H},(\mathbf{x})_{i}>0\right\}>0$ for some $i \in\{1, \ldots, n\}$;

(b) $(\mathcal{H}, B) \in$ Super $_{n}$ if and only if $\sup \left\{\frac{B(\mathbf{x})}{\max (\mathbf{x})} \mid \mathbf{x} \in \mathcal{H} \backslash\{0\}\right\}<\infty$.

Proof. For any $i \in\{1,2, \ldots, n\}$ let $\mathcal{H}_{i}$ be the set of all $\left.\left.z \in\right] 0,1\right]$ such that $(\mathbf{x})_{i}=z$ for some $\mathbf{x} \in \mathcal{H}$. Also, for any $i \in\{1,2, \ldots, n\}$ such that $\mathcal{H}_{i} \neq \emptyset$ and for every $z \in \mathcal{H}_{i}$ we let $B_{i}(z)=\inf \left\{B(\mathbf{x}) \mid \mathbf{x} \in \mathcal{H},(\mathbf{x})_{i}=z\right\}$. Now, for $(\mathrm{a})$, we have $(\mathcal{H}, B) \in S u b_{n}$ if and only if $B_{*}(\mathbf{1})>0$, which is equivalent to the existence of an $i \in\{1,2, \ldots, n\}$ such that $\left(B_{i}\right)_{*}(1)>0$. Since $B_{i}$ is a one-dimensional weighing function for $\mathcal{H}_{i}$, by part (a) of Proposition 4 the condition $\left(B_{i}\right)_{*}(1)>0$ is equivalent to $\inf \left\{B_{i}(x) / x \mid x \in \mathcal{H}_{i}\right\}>0$. By definition of $B_{i}$ the last condition is equivalent to $\inf \left\{B(\mathbf{x}) /(\mathbf{x})_{\mathbf{i}} \mid \mathbf{x} \in \mathcal{H},(\mathbf{x})_{\mathbf{i}}>\mathbf{0}\right\}>\mathbf{0}$, which proves (a).

For (b), let $\mathcal{H}_{0}$ be the set of all $\left.\left.z \in\right] 0,1\right]$ for which there exists an $\mathbf{x} \in \mathcal{H}$ such that $\max (\mathbf{x})=z$, and let $\tilde{B}(z)=\sup \{B(\mathbf{x}) \mid \max (\mathbf{x})=z\}$. We now have $(\mathcal{H}, B) \in \operatorname{Super}_{n}$ if and only if $B^{*}(\mathbf{1})<\infty$, which, by definition of $\tilde{B}$, happens if and only if $(\tilde{B})^{*}(1)<\infty$. Since $\tilde{B}$ is a one-dimensional weighing function for $\mathcal{H}_{0}$, by part (b) of Proposition 4 the condition $(\tilde{B})^{*}(1)<\infty$ is equivalent to $\sup \left\{\tilde{B}(x) / x \mid x \in \mathcal{H}_{0}\right\}<\infty$. Invoking the definition of $\tilde{B}$ again, the last condition is equivalent to $\sup \{B(\mathbf{x}) / \max (\mathbf{x}) \mid \mathbf{x} \in \mathcal{H}\}<\infty$, proving (b).

\section{$3 \quad$ Examples}

In this section we will present examples of functions $B_{*}$ and $B^{*}$ for specific decomposition sets and related decomposition weighting functions, as well as their links to some well known optimization and construction methods. 
Example 1 Let $\mathcal{H}=\{(0,0),(0.1,0.1)\}$ and $B(\mathbf{y})=y_{1}$, where $y_{1}$ is the first coordinate of y. Values of $B_{*}$ and $B^{*}$ are depicted in Figure 1 and Figure 2, respectively. Observe that $\sum_{i=1}^{k} \mathbf{y}^{(i)}$ appearing in expression (1) and (2) always have the form $k(0.1,0.1)$ for $k \in\{0,1, \ldots, 10\}$, because the only vector that can be used for summation is $(0.1,0.1)$. This explains the shape of the graphs in Figure 1 and Figure 2.

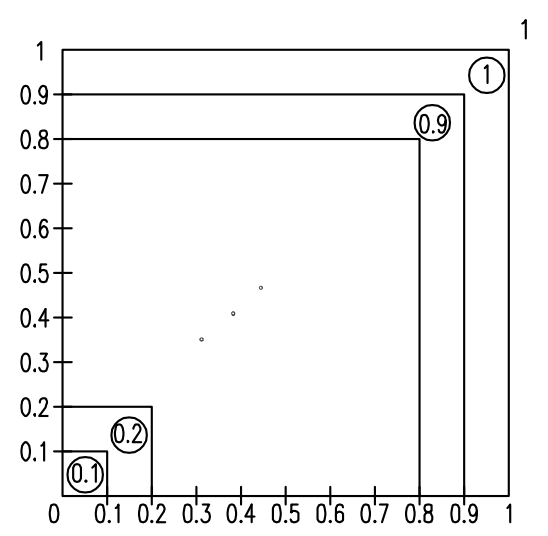

Figure 1: $B_{*}$ from Example 1

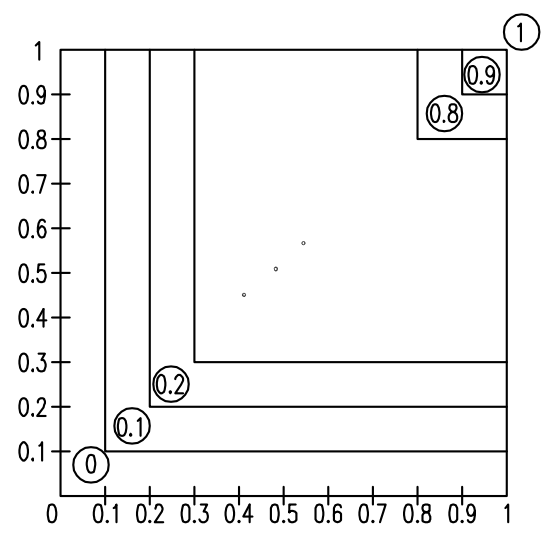

Figure 2: $B^{*}$ from Example 1

Example 2 Let $\mathcal{H}=\{(0,0),(0.8,0.3),(0.2,0.7)\}$ and let $B(0.8,0.3)=0.8, B(0.2,0.7)=$ 0.6 . It can be shown that in this case we have $B^{*}(1,1)=B_{*}(1,1)=1$.4. The corresponding values of $B_{*}$ and $B^{*}$ are depicted in Figure 3 and Figure 4 , respectively.

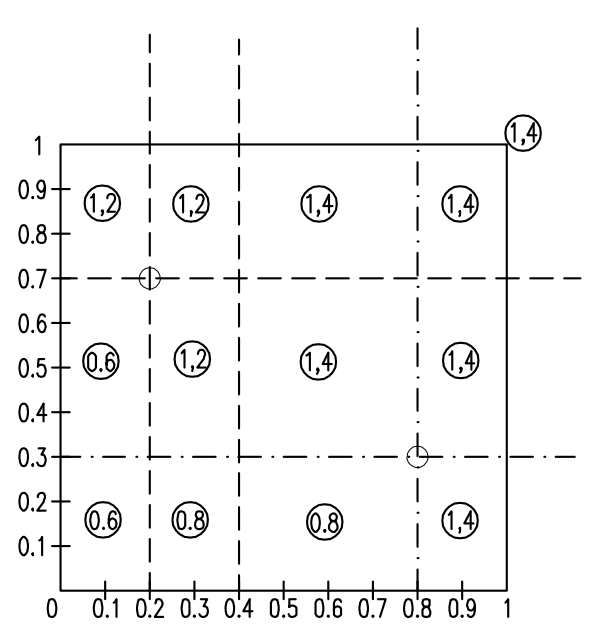

Figure 3: $B_{*}$ from Example 2

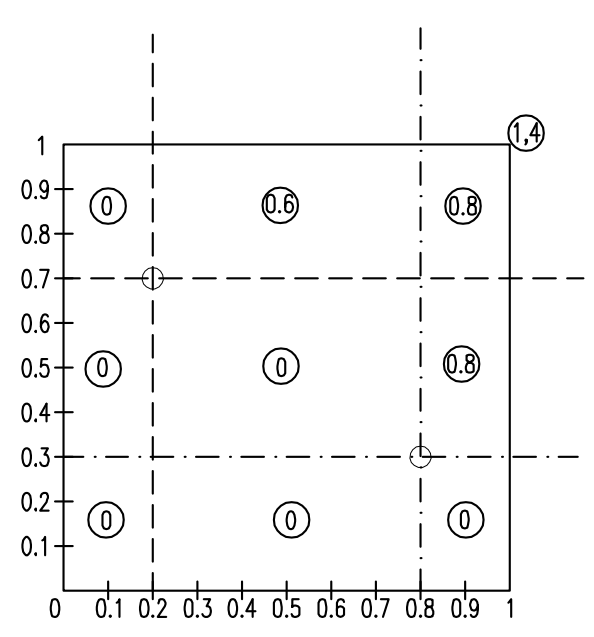

Figure 4: $B^{*}$ from Example 2 
Example 3 Let $\mathcal{H}=\{(0,0),(0.2,0.3),(0.5,0.7)\}$ and let $B=\prod$ be the product. $A$ schematic description of $B^{*}$ is in Figure 5.

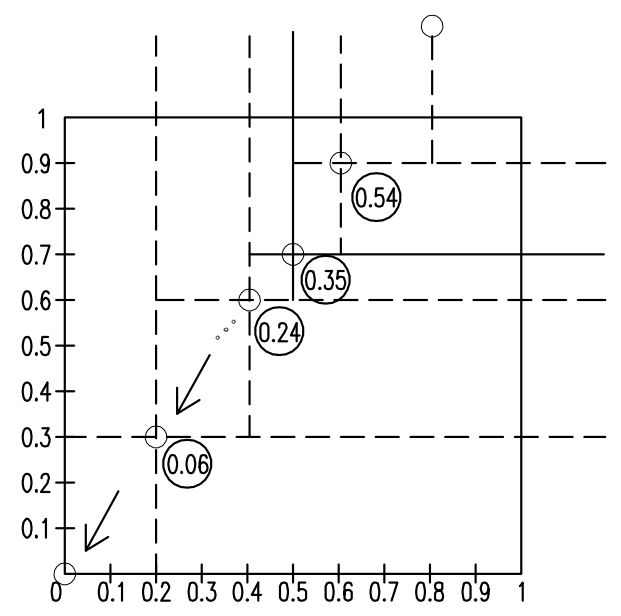

Figure 5: $B^{*}$ from Example 3

Example 4 In this example we will use a segment for $\mathcal{H}$ by letting $\mathcal{H}=\{(x, y) \mid x \in$ $[0.1,1], y=0.1-x\} \cup\{(0,0)\}$. The weighing function is defined as follows $B: \mathcal{H} \backslash\{(0,0)\} \rightarrow$ 0.05. The function $B^{*}$ is depicted in Figure 6 (2D) and in Figure 7 (3D).

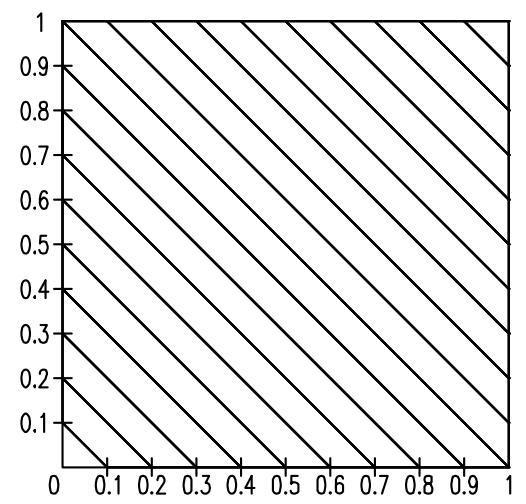

Figure 6: Contour lines of $B^{*}$ from Example 4

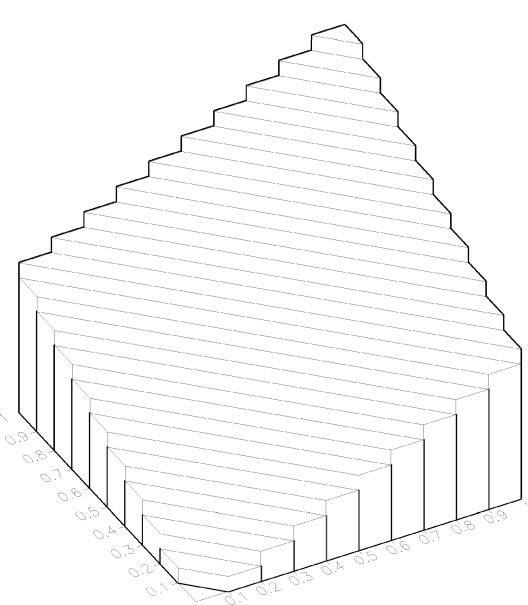

Figure 7: $3 D$ plot of $B^{*}$ from Example 4 
For a finite universe $X=\{1, \ldots, n\}$, any non-empty set $\mathscr{C} \subseteq 2^{X} \backslash\{\emptyset\}$ is called a collection, and any set $\mathcal{G}$ of collections is called a decomposition system. A set function $\mu: 2^{X} \rightarrow[0,1]$ which is monotone and satisfies the boundary conditions $\mu(\emptyset)=0$ and $\mu(X)=1$ is called a capacity. Lehrer in [9] introduced the decomposition integral $I_{\mathcal{G}, m}$ : $[0,1]^{n} \rightarrow[0, \infty]$ as follows:

$$
I_{\mathcal{G}, m}(\mathbf{x})=\sup \left\{\sum_{i=1}^{k} a_{i} \mu\left(E_{i}\right) \mid\left(E_{i}\right)_{i=1}^{k} \in \mathcal{G}, a_{1}, \ldots, a_{k} \geq 0, \sum_{i=1}^{k} a_{i} 1_{E_{i}} \leq \mathbf{x}\right\}
$$

Note that Lehrer's approach covers several types of integrals, including the Choquet integral [8] (when $\mathcal{G}$ consists from all maximal chains in $2^{X} \backslash\{\emptyset\}$ ), the Shilkret integral [12] (when $\mathcal{G}$ consists from all singleton collections), and PAN-integral [14], [15] (when $\mathcal{G}$ consists of all partitions of $X)$.

For a collection $\mathscr{C}=\left(E_{i}\right)_{i=1}^{k}$, denote $\mathcal{H}_{\mathscr{C}}=\left\{a_{i} 1_{E_{i}} \mid a_{i} \in[0,1], E_{i} \in \mathscr{C}\right\}$, and define $B_{\mathscr{C}, \mu}: \mathcal{H}_{\mathscr{C}} \rightarrow[0,1]$ by $B_{\mathscr{C}, \mu}\left(a_{i} 1_{E_{i}}\right)=a_{i} \mu\left(E_{i}\right)$. Evidently, $\mathcal{H}_{\mathscr{C}}$ is a decomposition set and $B_{\mathscr{C}, \mu}$ a weighing function, and thus $\left(B_{\mathscr{C}, \mu}\right)^{*}$ given by $(2)$ is well defined. Now, the next results are immediate.

Proposition 6 Let $\mathcal{G}=\left\{\mathscr{C}_{j} \mid j \in J\right\}$ be a decomposition system and $\mu$ a capacity on $X=\{1, \ldots, n\}$. Then $I_{\mathcal{G}, \mu}=\max \left\{\left(B_{\mathscr{C}_{j}, \mu}\right)^{*} \mid j \in J\right\}$, i.e., the decomposition integral is just the maximal value of newly introduced functionals $\left(B_{\mathscr{C}_{j}, \mu}\right)^{*}$.

Similarly, when considering the superdecomposition integral $I^{\mathcal{G}, \mu}$ introduced in [10] by

$$
I^{\mathcal{G}, m}(\mathbf{x})=\inf \left\{\sum_{i=1}^{k} a_{i} \mu\left(E_{i}\right) \mid\left(E_{i}\right)_{i=1}^{k} \in \mathcal{G}, a_{1}, \ldots, a_{k} \geq 0, \sum_{i=1}^{k} a_{i} 1_{E_{i}} \geq \mathbf{x}\right\}
$$

the next result is valid.

Proposition 7 Let $\mathcal{G}=\left\{\mathscr{C}_{j} \mid j \in J\right\}$ be a decomposition system and $\mu$ a capacity on $X=\{1, \ldots, n\}$. Then $I^{\mathcal{G}, \mu}=\min \left\{\left(B_{\mathscr{C}_{j}, \mu}\right)_{*} \mid j \in J\right\}$, i.e., the decomposition integral is just the minimal value of newly introduced functionals $\left(B_{\mathscr{C}_{j}, \mu}\right)_{*}$.

\section{Concluding remarks}

We have introduced two methods of constructing aggregation functions on $[0,1]$ in situation when only a partial information is available. We have exemplified the superadditive functions $B^{*}$ and the subadditive functions $B_{*}$, having in mind that the related aggregation functions $A^{\mathcal{H}, B}$ and $A_{\mathcal{H}, B}$ are easily obtained by normalization of $B^{*}$ and $B_{*}$, respectively. Besides this, we have shown the fact, that our approach can be seen as a generalization of decomposition and superdecomposition integrals [9], [10]. In a similar way, one can show

the fact, that the linear programming optimization problems can be formulated in the 
language of $B^{*}$ functionals (when we maximize the profit) and $B_{*}$ functionals (when we minimize the costs). Note that we can introduce weighing function $B$ and the decomposition set $\mathcal{H}$ related to $[0, \infty[$ interval instead of $[0,1]$ interval, and then no normalization is needed (compare also the Examples 1,2). More details about the links of the linear programming and $B^{*}, B_{*}$ functionals can be found in [13]. We expect applications of our approach in economics, social sciences, etc., and especially in multicriteria decision support.

Acknowledgement. The first, the second and the fifth author acknowledge support from the project VEGA 1/0420/15 and APVV 14-0013. The fifth author was supported also by the NPU II project LQ 1602. Research of the fourth author was supported by the "Programma Operativo Nazionale" Ricerca Competitività "2007-2013" within the project "PON04a2 E SINERGREEN-RES-NOVAE" and FIR Università di Catania, "New developments in Multiple Criteria Decision Aiding (MCDA) and their application to territorial competitiveness".

\section{References}

[1] G. Beliakov, A.Pradera, T.Calvo (2007). Aggregation Functions: A Guide for Practitioners. Springer, Berlin.

[2] B. Bensaid, J.P. Lesne, J.Scheinkman (1992). Derivative Asset Pricing with Transaction Costs1. Mathematical Finance, 2(2), 63-86.

[3] D. Darrell (2010). Dynamic asset pricing theory. Princeton University Press.

[4] M. Fuss, D. McFadden. (Eds.) (2014). Production Economics: A Dual Approach to Theory and Applications: Applications of the Theory of Production (Vol. 2). Elsevier.

[5] M. Grabisch, J-L.Marichal, R.Mesiar, E.Pap (2009). Aggregation Functions (Encyklopedia of Mathematics and its Applications). Cambridge University Press.

[6] S. Greco, R.Mesiar, F.Rindone, L.Šipeky (2014). The superadditive and the subadditive transformations of integrals and aggregation functions.

[7] S. Greco, R.Mesiar, F.Rindone, L.Šipeky (2016). Decomposition approaches to integration without a measure.Fuzzy sets and Systems, to appear, doi:10.1016/j.fss.2015.07.014.

[8] G. Choqet (1953). Theory of capacities, Ann. Inst. Fourier (5), p.131-295

[9] E. Lehrer (2009). A new integral for capacities. Economic Theory, Springer, vol. 39 (1), p. $157-176$ 
[10] R. Mesiar, J. Li, E.Pap (2015) Superdecomposition integrals. Fuzzy Sets and Systems 259 , p. $3-11$

[11] R.W. Shepherd (2015). Theory of cost and production functions. Princeton University Press.

[12] N. Shilkret (1971) Maxitive measure and integration. In Indagationes Mathematicae (Proceedings), volume 74, pages 109-116. Elsevier

[13] L. Šipeky, A. Šipošová (2015) Constraint super- and sub-additive transformations. 3 p. Proc. Conf. CITCEP 2015, Krakow, to appear.

[14] Q. Yang (1985) The Pan-integral on the Fuzzy Measure Space, Fuzzy Mathematics 3, $107-114$.

[15] Z. Wang, G.J.Klir (2009) Generalized Measure Theory. Springer, New York. 